Revista Brasileira de Agricultura Irrigada v.12, nº.7, p. 3146 - 3158, 2018

ISSN 1982-7679 (On-line)

Fortaleza, CE, INOVAGRI - http://www.inovagri.org.br

DOI: $10.7127 /$ rbai.v12n7001027

Protocolo 1027.18 - 05/10/2018 Aprovado em 28/02/2019

\title{
ESTIMATIVA DA RADIAÇÃO SOLAR GLOBAL PARA A REGIÃO FRONTEIRA OESTE DO RIO GRANDE DO SUL
}

Elisa de Almeida Gollo ${ }^{1}$, Adroaldo Dias Robaina ${ }^{2}$, Marcia Xavier Peiter ${ }^{2}$, Miguel Chaiben Neto ${ }^{3}$, Jhosefe Bruning ${ }^{1}$, Francielle Altíssimo Bortolás ${ }^{3}$

\begin{abstract}
RESUMO
O objetivo do presente trabalho foi avaliar e calibrar diferentes modelos de estimativa da radiação solar global para três municípios da região Fronteira Oeste do Rio Grande do Sul. Para isso, foram utilizados dados médios diários de temperatura máxima e mínima do ar e radiação solar, de estações automáticas do Instituto Nacional de Meteorologia (INMET), localizadas nos municípios de Alegrete, Uruguaiana e São Borja. A radiação solar global estimada foi obtida através da utilização de dez modelos baseados na amplitude térmica diária e destes, quatro foram calibrados a partir da série histórica de dados para cada município. A análise de regressão linear simples e outros parâmetros de desempenho foram utilizados para a avaliação dos modelos. Os modelos de Ball et al. (2004) e Chen et al. (2004), com os coeficientes calibrados nesse estudo, apresentaram a melhor estimativa da radiação solar global para a região Fronteira Oeste do Rio Grande do Sul, seguidos pelos modelos de Annandale et al. (2002) e Goodim et al. (1999). Já os modelos Weiss et al. (2001), Thornton \& Running, (1999), Donatelli e Campbell (1998), Richardson (1985) e Bristow e Campbel (1984), não são recomendados para a região Fronteira Oeste do Rio Grande do Sul.
\end{abstract}

Palavras-chave: modelos de estimativa da radiação, amplitude térmica, equações empíricas, calibração de coeficientes

\section{ESTIMATION OF GLOBAL SOLAR RADIATION FOR THE WESTERN BORDER OF RIO GRANDE DO SUL}

\begin{abstract}
The objective of the present work was to evaluate and calibrate different models of estimation of global solar radiation for three cities in the West Border region of Rio Grande do Sul. For this purpose, daily average data of maximum and minimum temperature of air and solar radiation, of automatic stations of the Instituto Nacional de Meteorologia (INMET), located in Alegrete, Uruguaiana and São Borja. Global solar radiation was obtained using ten estimation models based on the daily thermal amplitude

\footnotetext{
${ }^{1}$ Doutorando em Engenharia Agrícola, Universidade Federal de Santa Maria, e-mail: elisagollo@hotmail.com; jhosefe.b@gmail.com

${ }^{2}$ Doutor em Engenharia Civil, Professor da Universidade Federal de Santa Maria fbortolas@gmail.com
}

${ }^{3}$ Mestrando em Engenharia Agrícola, Universidade Federal de Santa Maria, e-mail: michelchainem@ gmail.com;
\end{abstract}


and of these, four were calibrated from the historical series of global solar radiation data for each city. The simple linear regression analysis and other performance parameters were used to evaluate the models. The models of Ball et al. (2004) and Chen et al. (2004), with the coefficients calibrated in this study, presented the best estimate of global solar radiation for the western border region of Rio Grande do Sul, followed by the models of Annandale et al. (2002) and Goodim et al. (1999). The Weiss et al. (2001), Thornton and Running, (1999), Donatelli and Campbell (1998), Richardson (1985) and Bristow and Campbel (1984) are not recommended for this region.

Keywords: radiation estimation models, thermal amplitude, empirical equations, calibration of coefficients.

\section{INTRODUÇÃO}

O estado do Rio Grande do Sul é responsável por $69 \%$ da produção nacional de arroz, sendo cultivados, aproximadamente, 1,1 milhões de hectares ao ano (CONAB, 2018). Segundo dados do IRGA (2018), a produção total no estado para a safra $2016 / 17$ foi de 8,2 milhões de toneladas. A Fronteira Oeste, conforme Beutler et al. (2012), caracteriza-se como a região mais importante em volume de produção do Estado, sendo os municípios de Uruguaiana, Itaqui, Alegrete e São Borja, destaque na produção, responsáveis por $31 \%$ da produção estadual (IRGA, 2018).

Um dos fatores que justificam a produção da região Fronteira Oeste é a condição climática favorável, devido à alta temperatura do ar e aos elevados índices de radiação solar incidente no período de cultivo (ALSALAYMEH, 2006; STEINMETZ et al., 2003). Devido ao sistema de cultivo inundado adotado no estado para o arroz, essa cultura recebe adequado suprimento hídrico durante a quase totalidade de seu período de cultivo, sendo a produtividade de grãos fortemente influenciada pela temperatura do ar e disponibilidade de radiação solar (STEINMETZ et al., 2013).

Por esse motivo, em alguns anos, reduções no rendimento de grãos de arroz são observadas no estado, devido, muitas vezes, a ocorrência de condições climáticas desfavoráveis durante o cultivo, principalmente menores temperatura do ar e radiação solar (STEINMETZ; BRAGA, 2001).

Modelos de simulação do desenvolvimento e produção do arroz são ferramentas de uso crescente que, através de previsões para anos desfavoráveis climaticamente, auxiliam no planejamento das safras, minimizando prejuízos de produção. Nesse sentido, a quantificação da radiação solar, por ser um dado de entrada aos modelos, é necessária para a utilização de modelagem nas previsões de produção, pois esta varia de acordo com as condições atmosféricas e latitude de cada local (ALMOROX et al., 2011).

Segundo Buriol et al. (2012), a obtenção de dados de radiação solar global para determinados locais é limitada, principalmente, pelo fato de que poucas estações meteorológicas possuem registros de radiação solar global, devido ao elevado custo e frequentes manutenções pelas quais os aparelhos utilizados precisam passar.

Como alternativa, para a obtenção da radiação solar global, modelos matemáticos de estimativa podem ser utilizados. Segundo Benghanem e Mellit (2014) existe grande variabilidade de modelos desenvolvidos, usando relações empíricas, para estimar a radiação solar global ao nível do solo a partir da diferença entre temperaturas extremas do ar.

Porém, a precisão desses modelos precisa ser verificada para cada local considerado, pois, de acordo com Borges et al. (2010), esses modelos, em geral, são restritos às condições para as quais foram desenvolvidos, apresentando problemas de transferabilidade, quando não devidamente testados e calibrados.

Em vista do exposto, o objetivo do presente trabalho foi avaliar e calibrar diferentes modelos de estimativa da radiação solar global para três municípios da região Fronteira Oeste do Rio Grande do Sul. 


\section{MATERIAL E MÉTODOS}

Para a realização do presente trabalho, foram utilizados dados médios diários de temperatura máxima e mínima do ar $\left({ }^{\circ} \mathrm{C}\right)$ e radiação solar $\left(\mathrm{MJ} \mathrm{m} \mathrm{m}^{-2} \mathrm{dia}^{-1}\right)$, da rede de estações automáticas do Instituto Nacional de Meteorologia (INMET), localizadas em três municípios da região Fronteira Oeste do estado do Rio Grande do Sul (Tabela 1), que possuíam série histórica de dados disponível. O clima da região, segundo a classificação de Köeppen é do tipo cfa-subtropical úmido, sem estação seca e com temperatura média de $14,3^{\circ} \mathrm{C}$ no inverno e $26,3^{\circ} \mathrm{C}$ no verão, com precipitação anual média de $1400 \mathrm{~mm}$ (ALVARES et al., 2013).

Tabela 1. Municípios estudados, códigos OMM, coordenadas geográficas das estações meteorológicas e período estudado.

\begin{tabular}{cccccc}
\hline \multirow{2}{*}{ Município } & Código & \multicolumn{3}{c}{ Coordenadas geográficas } & \multirow{2}{*}{ Período } \\
\cline { 3 - 5 } & OMM & Latitude & Longitude & Altitude $(\mathrm{m})$ & \\
\hline Alegrete & 86975 & $-29,42$ & $-55,31$ & 121 & $2007-2016$ \\
São Borja & 86960 & $-28,38$ & $-56,0$ & 83 & $2008-2016$ \\
Uruguaiana & 86973 & $-29,50$ & $-57,04$ & 62,3 & $2007-2016$ \\
\hline
\end{tabular}

Os dados horários de temperatura obtidos através das estações foram transformados em diários e a radiação solar considerada neste estudo foi a total acumulada durante o dia. Para a estimativa da radiação solar global, foram utilizados dez modelos de estimativa baseados na amplitude térmica diária (Tabela 2). Todos os modelos utilizados também requerem dados de radiação solar no topo da atmosfera $(\mathrm{Ra})$. A mesma foi calculada através do método proposto por Allen et al. (1998), conforme a Eq. 1; Eq. 2; Eq. 3 e Eq. 4.

$$
\begin{aligned}
& \mathrm{Ra}=\frac{24 * 60}{\pi} * \mathrm{G}_{\mathrm{sc}} * \mathrm{~d}_{\mathrm{r}} *\left(\omega_{\mathrm{s}} * \operatorname{sen} \varphi * \operatorname{sen} \delta+\cos \varphi * \cos \delta * \operatorname{sen} \omega_{\mathrm{s}}\right) \\
& \mathrm{d}_{\mathrm{r}}=1+0,033 * \cos \left(\frac{2 \pi * J}{365}\right) \\
& \omega_{\mathrm{s}}=\cos ^{-1}(-\tan \varphi * \tan \delta) \\
& \delta=0,409 * \operatorname{sen}\left(\frac{2 \pi * J}{365}-1,39\right)
\end{aligned}
$$

Em que: $\mathrm{G}_{\mathrm{sc}}$ é constante solar $\left(0,0820 \mathrm{MJ} \cdot \mathrm{m}^{-2} \cdot \mathrm{min}^{-1}\right) ; \mathrm{d}_{\mathrm{r}}$ é distância relativa entre a Terra e o Sol; $\omega_{\mathrm{s}}$ é ângulo horário do pôr do Sol, em radianos; $\varphi$ é latitude do local, em radianos; $\delta$ é declinação solar, em radianos; J é dia juliano.

Os modelos de Ball et al. (2004), Chen et al. (2004), Richardson (1985) e Bristow e Campbel (1984), requerem a utilização dos coeficientes "a", "b" e "c" em seus modelos, os quais foram calibrados a partir da série histórica de dados de radiação solar global para cada município conforme a Tabela 1 . A validação dos dados não foi realizada.
A análise de regressão linear simples, através do modelo $\mathrm{Yi}=\mathrm{a}+\mathrm{b} \hat{\mathrm{Y}} \mathrm{i}$, para a comparação dos valores de Rs obtidos pelas estações meteorológicas (Yi) e pelos dez modelos de estimativa utilizados (Ŷi), foi utilizada a fim de estabelecer os modelos que melhor estimam a Rs em relação aos dados observados. 
Tabela 2. Modelos: Ball et al. (2004), Chen et al. (2004), Annandale et al. (2002), Weiss et al. (2001), Goodim et al. (1999), Thornton e Running, (1999), Donatelli e Campbell (1998), Richardson (1985), Bristow e Campbel (1984) e Hargreaves e Samani (1982); utilizados para estimar a radiação solar global, abreviaturas e suas equações.

\begin{tabular}{lccc}
\multicolumn{1}{c}{ Autores } & Abrev & Equações & $\mathrm{N}^{\circ}$ da \\
Equação
\end{tabular}

Alt = altitude; "a", "b" e "c"= coeficientes; $\mathrm{K}_{\mathrm{Rs}}=$ coeficiente empírico (0,16 para cidades do interior e 0,19 para cidades litorâneas); $\mathrm{T}_{\mathrm{tmax}}=$ transmissividade máxima ocorrida em um dia de céu claro; $\mathrm{T}_{\mathrm{tf}}=$ é uma fração de $\mathrm{T}_{\mathrm{tmax}}$.

Para avaliar o desempenho dos modelos foram utilizados os coeficientes de determinação $\left(\mathrm{R}^{2}\right)$ obtidos através das análises de regressão matemática. Foram considerados como de melhor desempenho os métodos com coeficiente de determinação mais próximos a um. Também foram utilizados a raiz do quadrado médio do erro (RQME), o erro absoluto médio (EAM), o índice de Willmott (d) e o índice de Camargo e Sentelhas (c), conforme a Tabela 3, para avaliar o desempenho dos modelos. Para os indicadores "RQME" e "EAM" valores próximos a zero indicam o modelo mais adequado.

Já o índice "c" qualifica os modelos de acordo com o seu desempenho, sendo os melhores aqueles que obtiverem valores próximos a um (Tabela 4).

Tabela 3. Indicadores de desempenho, raiz do quadrado médio do erro (RQME), erro absoluto médio (EAM), índice de Willmott (d) e índice de Camargo e Sentelhas (c) utilizados para classificar os modelos quanto a estimativa da radiação global.
Parâmetros de
desempenho
Equações
$N^{o}$ da Equação 


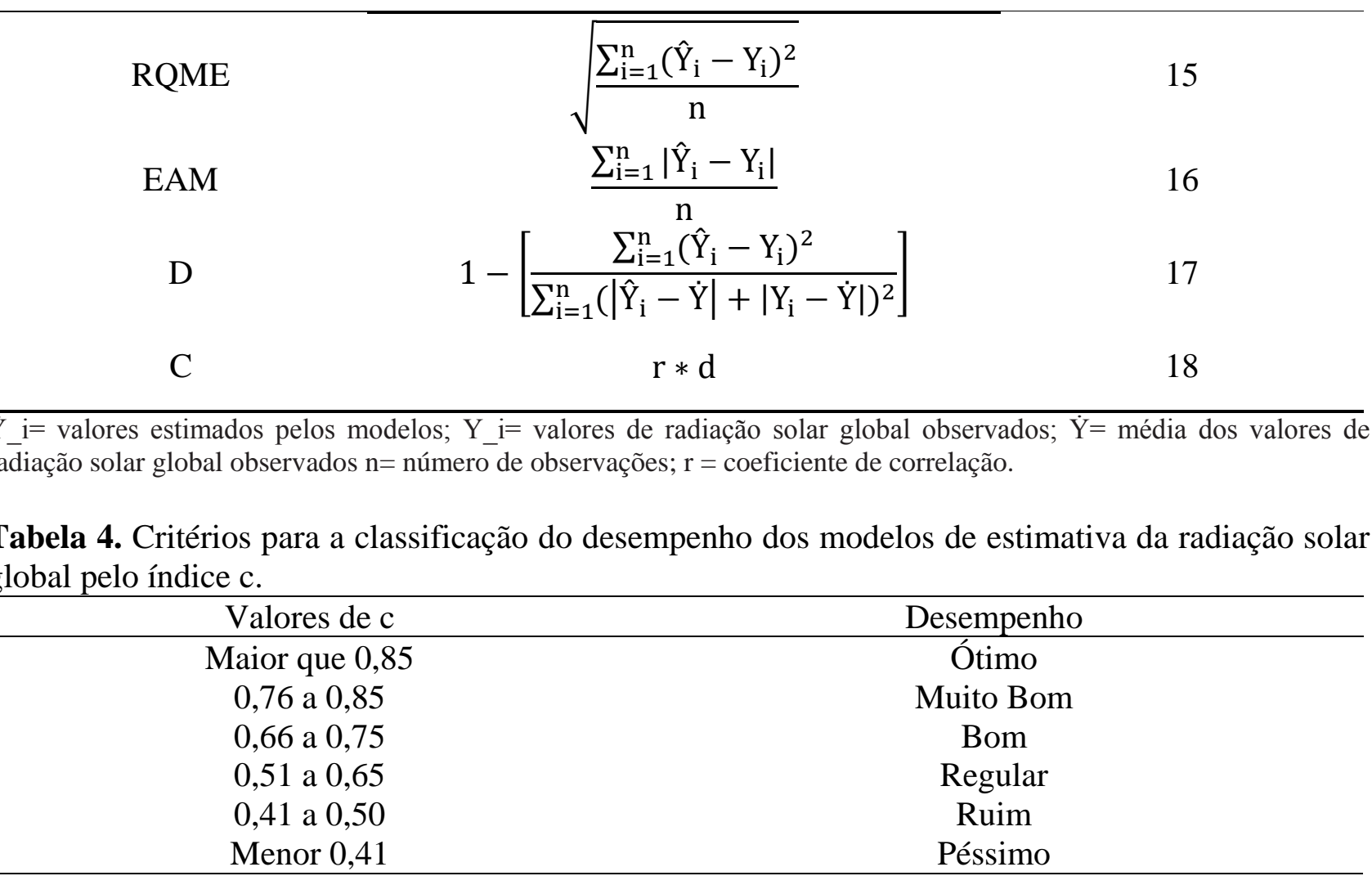

Fonte: Adaptado de Camargo e Sentelhas, (1997).

\section{RESULTADOS E DISCUSSÃO}

Dos dez modelos de estimativa da radiação solar global (Rs) estudados, foi realizada a calibração em quatro, que foram:
Ball et al. (2004) (Ball), Chen et al. (2004) (Chen), Richardson (1985) (HS-R) e Bristow e Campbel (1984) (BC); os valores dos coeficientes calibrados podem ser observados na Tabela 5 .

Tabela 5. Valores dos coeficientes de calibração os modelos de estimativa da radiação solar global de Ball et al. (2004) (Ball), Chen et al. (2004) (Chen), Richardson (1985) (HS-R) e Bristow e Campbel (1984) (BC), para os municípios de Alegrete, Uruguaiana e São Borja, para o período estudado.

\begin{tabular}{cccc}
\hline \multirow{2}{*}{ Modelos } & \multicolumn{3}{c}{ Coeficientes } \\
\cline { 2 - 4 } & & $\mathrm{b}$ & $\mathrm{C}$ \\
Ball & 0,09 & Alegrete & \\
Chen & 0,09 & 1,52 & \\
HS-R & 0,28 & 0,76 & 2,05 \\
BC & & 0,25 & \\
& & & \\
Ball & 0,08 & Uruguaiana \\
Chen & 0,08 & 1,56 & 2,05 \\
HS-R & 0,31 & 0,26 & \\
BC & & & \\
Ball & 0,15 & São Borja
\end{tabular}




$\begin{array}{ccc}\text { Chen } & 0,15 & 0,68 \\ \text { HS-R } & 0,21 & 0,30 \\ \text { BC } & & \end{array}$

Os coeficientes $\mathrm{a}, \mathrm{b}$ e c apresentaram pouca ou nenhuma variação para Alegrete e Uruguaiana, o que provavelmente ocorreu devido a esses municípios apresentarem latitude muito próxima. Resultado semelhante foi obtido por Macêdo et al. (2016), que também encontrou pouca variação entre o coeficiente c de Bristow e Campbell (1984) para municípios próximos, constatando a calibração desse coeficiente não é necessária em locais semelhantes.

Já para São Borja, observou-se uma sensível variação dos coeficientes calibrados em relação aos outros dois municípios estudados e supracitados. Além de diferenças na topografia e altitude em relação aos municípios de Alegrete e Uruguaiana, essa variação deve estar relacionada, provavelmente, à proximidade da estação meteorológica desse município ao rio Uruguai. A presença de vapor d'água na atmosfera influência diretamente na radiação global alterando sua emitância, e a magnitude com que é irradiada. Dessa forma, a água presente na atmosfera aumenta a nebulosidade, fator que também minimiza a amplitude térmica no local, o que altera as características da atmosfera, e pode limitar a aplicabilidade de alguns modelos (DAL PAI; ESCOBEDO, 2015).

Os parâmetros de desempenho dos modelos podem ser observados na Tabela 6 . Para os três municípios, observou-se considerável variação de desempenho entre os dez modelos estudados. Observa-se que os modelos de Ball e Chen, de acordo com todos os parâmetros, foram os que obtiveram o melhor desempenho, entre os demais estudados, para Alegrete e Uruguaiana. Esse resultado corrobora com o encontrado por Macêdo et al. (2016), em seu estudo realizado para seis cidades do estado do Ceará, que também encontraram os modelos de Ball e Chen entre os melhores avaliados. Já para São Borja, os modelos GD e BC juntamente com os modelos supracitados, apresentaram os melhores desempenhos.

Tabela 6. Coeficiente de determinação da análise de regressão da $\mathrm{Rs}\left(\mathrm{R}^{2}\right)$, raiz do quadrado médio do erro (RQME), erro absoluto médio (EAM), índice de Willmott (d) índice de Camargo e Sentelhas (c) e desempenho, obtidos através das comparações entre a radiação solar global (Rs) estimada pelos diferentes modelos em comparação com os valores de observados obtidos através das estações meteorológicas, para os municípios de Alegrete, São Borja e Uruguaiana, para o período de estudo.

\begin{tabular}{lcccccc}
\hline \multirow{2}{*}{ Modelos } & \multicolumn{7}{c}{ Parâmetros de desempenho } \\
\cline { 2 - 7 } & $\mathrm{R}^{\mathbf{2}}$ & RQME & EAM & $\mathrm{d}$ & $\mathrm{c}$ & Classificação \\
\hline \multirow{2}{*}{ Ball } & 0,71 & 4,79 & 3,65 & 0,90 & 0,76 & Muito Bom \\
Chen & 0,71 & 4,81 & 3,68 & 0,90 & 0,76 & Muito Bom \\
Ann & 0,69 & 5,12 & 4,05 & 0,87 & 0,73 & Bom \\
Ws & 0,59 & 7,03 & 5,45 & 0,77 & 0,59 & Regular \\
GD & 0,66 & 5,72 & 3,89 & 0,87 & 0,71 & Bom \\
TR & 0,57 & 12,35 & 10,75 & 0,67 & 0,50 & Ruim \\
DC & 0,41 & 9,57 & 6,72 & 0,70 & 0,45 & Ruim \\
HS-R & 0,71 & 19,28 & 18,06 & 0,55 & 0,47 & Ruim \\
BC & 0,64 & 5,44 & 4,22 & 0,86 & 0,69 & Bom \\
HS & 0,69 & 5,13 & 4,07 & 0,87 & 0,73 & Bom \\
\hline & & \multicolumn{7}{c}{ Uruguaiana } \\
Ball & 0,74 & 4,45 & 3,33 & 0,92 & 0,79 & Muito Bom
\end{tabular}




\begin{tabular}{lcccccc} 
Chen & 0,74 & 4,45 & 3,33 & 0,92 & 0,79 & Muito Bom \\
Ann & 0,72 & 4,84 & 3,87 & 0,89 & 0,75 & Bom \\
Ws & 0,62 & 6,88 & 5,31 & 0,77 & 0,61 & Regular \\
GD & 0,68 & 5,35 & 3,73 & 0,88 & 0,73 & Bom \\
TR & 0,60 & 11,85 & 10,36 & 0,68 & 0,53 & Regular \\
DC & 0,46 & 8,90 & 6,20 & 0,72 & 0,49 & Ruim \\
HS-R & 0,74 & 20,86 & 19,69 & 0,52 & 0,45 & Ruim \\
BC & 0,67 & 5,12 & 4,03 & 0,87 & 0,71 & Bom \\
HS & 0,72 & 4,85 & 3,88 & 0,88 & 0,75 & Bom \\
\hline & & & & São Borja & & \\
Ball & 0,64 & 5,11 & 3,87 & 0,87 & 0,70 & Bom \\
Chen & 0,64 & 5,11 & 3,87 & 0,87 & 0,70 & Bom \\
Ann & 0,63 & 7,30 & 6,18 & 0,71 & 0,57 & Regular \\
Ws & 0,20 & 13,71 & 11,74 & 0,51 & 0,22 & Péssimo \\
GD & 0,65 & 5,67 & 4,45 & 0,86 & 0,69 & Bom \\
TR & 0,61 & 9,27 & 7,55 & 0,74 & 0,58 & Regular \\
DC & 0,51 & 8,58 & 6,14 & 0,74 & 0,53 & Regular \\
HS-R & 0,64 & 14,74 & 13,44 & 0,61 & 0,49 & Ruim \\
BC & 0,65 & 5,01 & 3,65 & 0,89 & 0,72 & Bom \\
HS & 0,63 & 7,32 & 6,20 & 0,71 & 0,57 & Regular \\
\hline
\end{tabular}

Os modelos de Ball e Chen apresentaram desempenho classificado como muito bom, de acordo com o índice c de Camargo e Sentelhas (1997) para Alegrete e Uruguaiana e classificado como bom para São Borja. Em relação ao erro absoluto médio (EAM) foram observados os valores de 3,65, 3,33 e 3,87 MJ $\mathrm{m}^{-2}$ dia $^{-1}$ para o modelo de Ball e 3,68, 3,33 e 3,87 MJ.m $\mathrm{m}^{-2} \cdot \mathrm{dia}^{-1}$ para o modelo de Chen para os municípios de Alegrete, Uruguaiana e São Borja respectivamente. Os modelos GD e BC, que juntamente com os modelos supracitados obtiveram melhor desempenho para São Borja, apresentaram classificação, conforme índice c, bom, e EAM de 4,45 e 3,65 MJ.m ${ }^{-2} \cdot \mathrm{dia}^{-1}$, respectivamente.

Para o indicador de desempenho raiz quadrada média do erro (RQME) os modelos de Ball et al. (2004) e Chen et al. (2004) obtiveram o melhor desempenho para os munícipios de Alegrete e Uruguaiana, para o munícipio de São Borja o modelo Bristow e Campbel (1984) obteve o melhor desempenho 5,01 seguido por Ball et al. (2004) e Chen et al. (2004) que obtiveram 5,11.
A partir da análise dos demais modelos, observou-se o desempenho satisfatório dos modelos Ann, GD, BC e HS para os municípios de Alegrete e Uruguaiana, sendo classificados como bom, com EAM variando entre 3,73 a 4,07 MJ.m ${ }^{-2} \cdot \mathrm{dia}^{-1}$, RQME de 4,84 a 5,72 e índice d de Willmott entre 0,86 e 0,89 . Souza et al. (2017), estudando os quatro modelos supracitados para o estado do Mato Grosso, obtiveram variação do índice d aproximado com os encontrados nesse trabalho, variando entre 0,80 e 0,90, porém, com menores valores de RQME, variando entre 1,52 e 4,58 entre os modelos. Para São Borja os modelos Ann e HS não obtiveram o mesmo desempenho que para os outros dois municípios, sendo classificados como regular.

Os modelos WS, DC, HR-S, TR obtiveram desempenho variando entre regular, ruim e péssimo para os três municípios estudados, apresentando EAMs que chegam a 19,69 MJ.m ${ }^{-2} \cdot \mathrm{dia}^{-1}$. Devido ao baixo desempenho, a utilização desses modelos para estimativa da Rs para a Fronteira Oeste deve ser desestimulada. 
As Figuras 1, 2 e 3 apresentam as representações gráficas da radiação solar global (Rs) observada através dos dados das estações meteorológicas, comparada aos valores de Rs estimados pelos modelos estudados para os municípios da Fronteira Oeste Alegrete, Uruguaiana e São Borja. Observa-se que a dispersão dos pontos em torno da linha de regressão e da reta 1:1 reforça os parâmetros de desempenho apresentados na Tabela 6 .
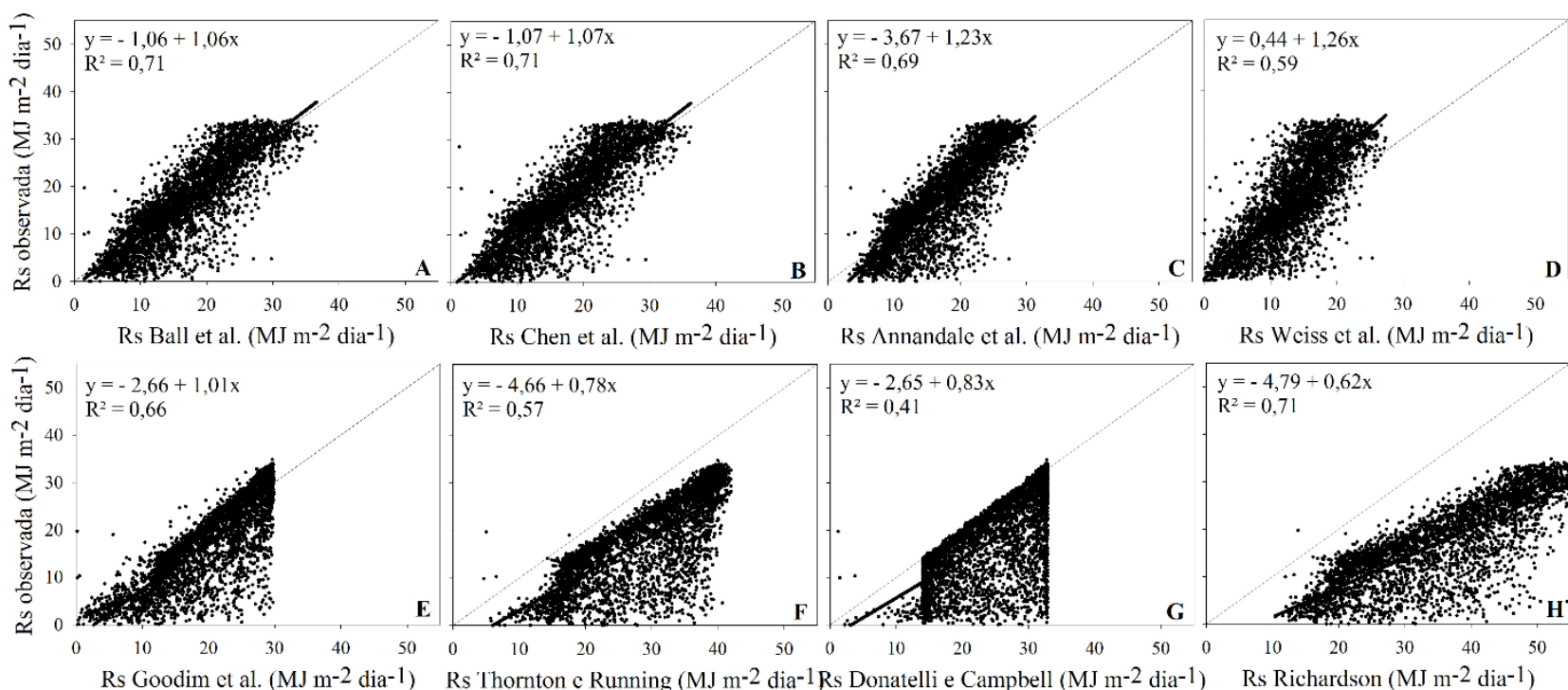

Rs $\Lambda$ nnandale et al. (MJ m-2 dia-1)
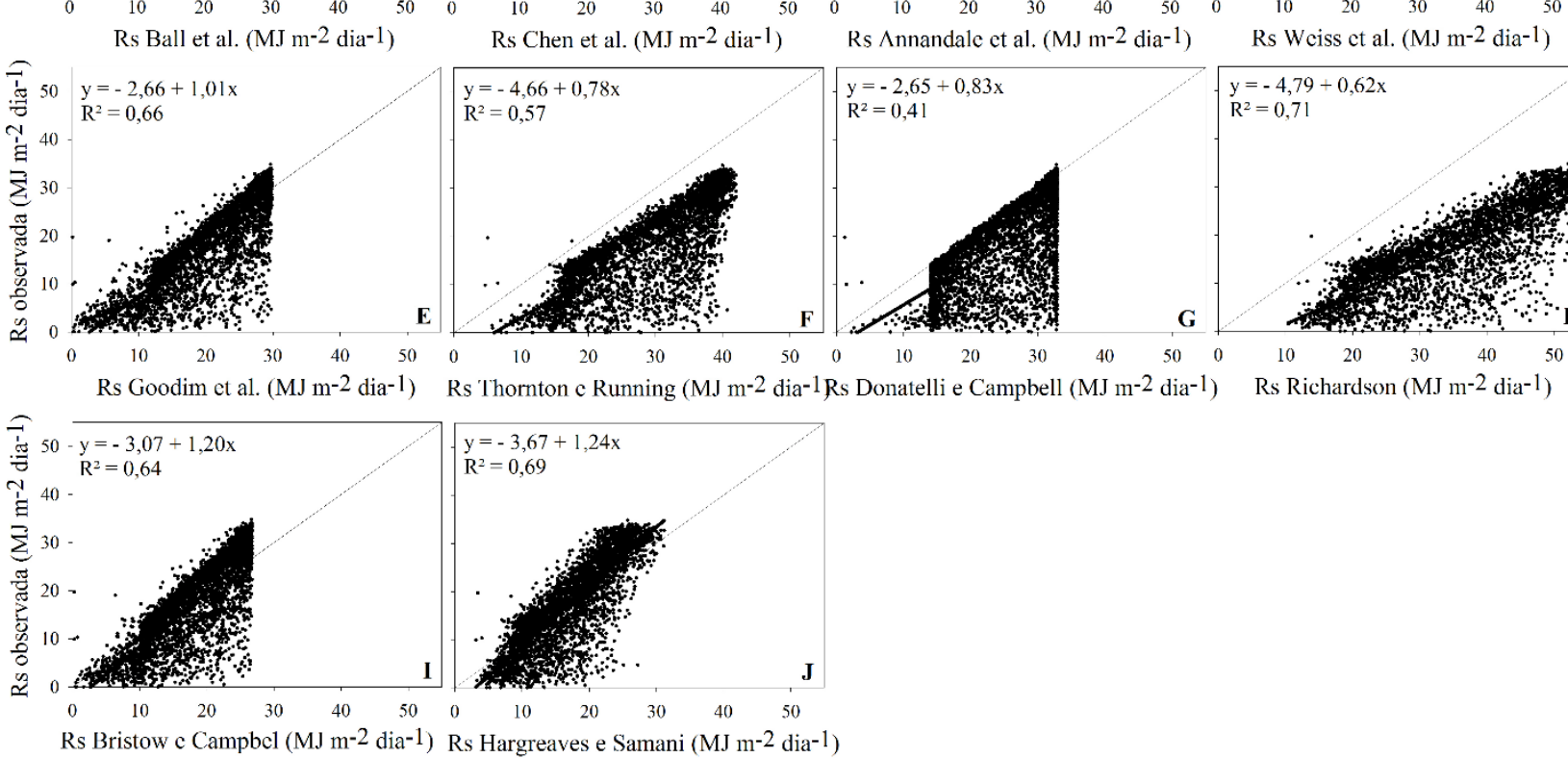

Figura 1. Representação gráfica da estimativa da radiação solar global (Rs) observada através dos dados da estação meteorológica, comparada com os valores de Rs obtidos pelos modelos de Ball et al. (2004) (1A), Chen et al. (2004) (1B), Annandale et al. (2002) (1C), Weiss et al. (2001) (1D), Goodim et al. (1999) (1E), Thornton e Running, (1999) (1F), Donatelli e Campbell (1998) (1G), Richardson (1985) (1H), Bristow e Campbel (1984) (1I) e Hargreaves e Samani (1982) (1J), para o município de Alegrete-RS, para o período de 2007 a 2016.

A dispersão dos pontos em torno da linha de tendência e da reta 1:1 para os municípios de Alegrete (Figura 1) e Uruguaiana (Figura 2) apresentaram comportamentos semelhantes para todos os modelos, sendo os modelos de Ball et al. (2004), Chen et al. (2004), Annandele et al. (2002) e Hargreaves e Samani (1982) aqueles apresentaram o melhor ajuste dos pontos na estimativa da radiação global. Apesar de apresentarem uma pequena tendência de subestimar a Rs, esses modelos foram os que mais se aproximaram da reta de referência $1: 1$, apresentando coeficientes de correlação $\left(\mathrm{R}^{2}\right)$ variando entre 0,69 e 0,74 . 

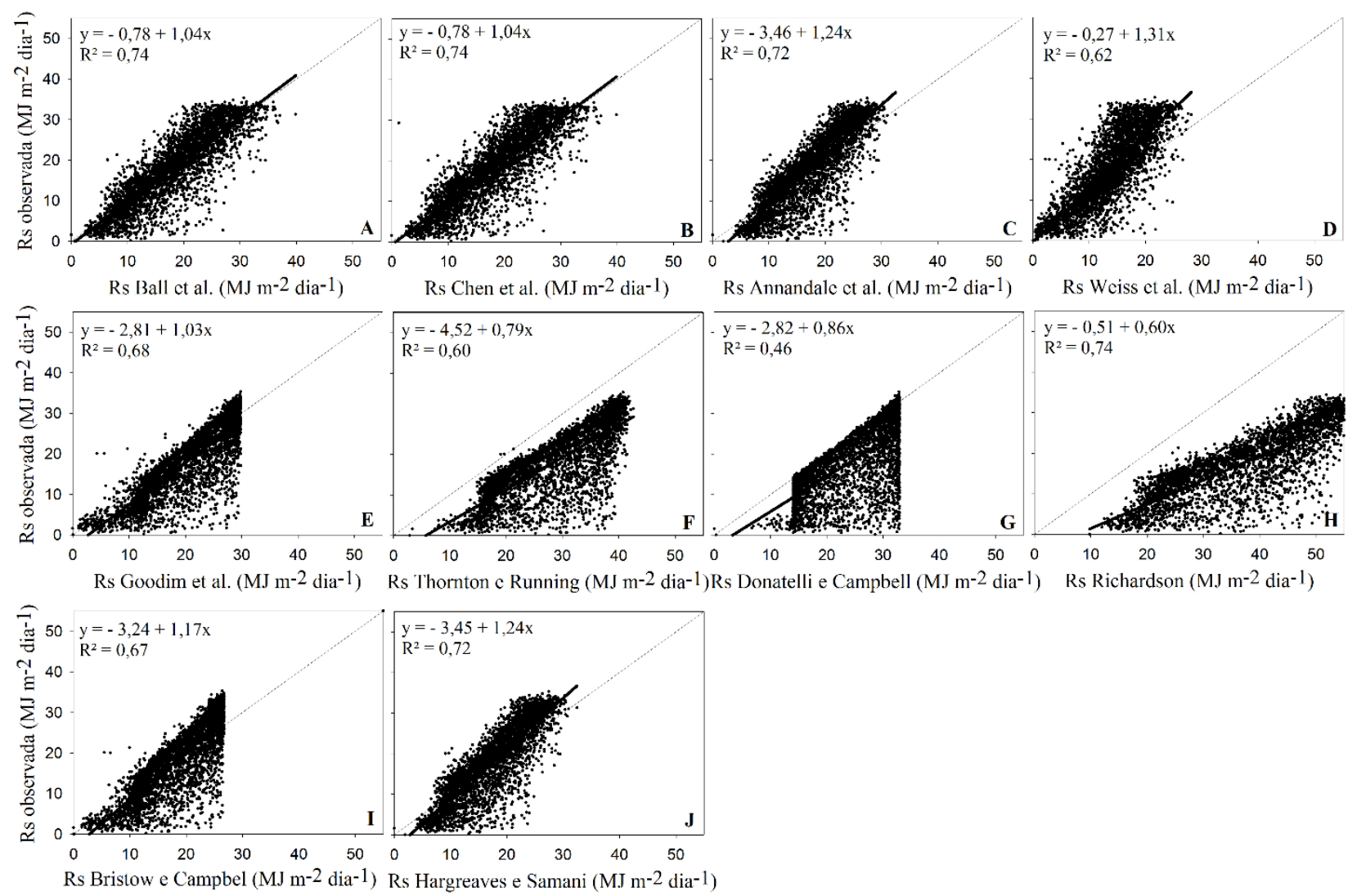

Figura 2. Representação gráfica da estimativa da radiação solar global (Rs) observada através dos dados da estação meteorológica, comparada com os valores de Rs obtidos pelos modelos de Ball et al. (2004) (1A), Chen et al. (2004) (1B), Annandale et al. (2002) (1C), Weiss et al. (2001) (1D), Goodim et al. (1999) (1E), Thornton e Running, (1999) (1F), Donatelli e Campbell (1998) (1G), Richardson (1985) (1H), Bristow e Campbel (1984) (1I) e Hargreaves e Samani (1982) (1J), para o município de Uruguaiana-RS, para o período de 2007 a 2016.

Ainda se observa nas Figuras 1 e 2 observou-se que o modelo proposto por Weiss et al. (2001) apresentou tendência de subestimar a Rs, apresentando $\mathrm{R}^{2}$ de 0,59 e 0,62 para Alegrete e Uruguaiana, respectivamente. Já os modelos de Thornton e Running, (1999), Donatelli e Campbell (1998) e Richardson (1985) apresentaram tendência de superestimar a Rs, concentrando os pontos abaixo da reta 1:1.
Para os modelos de Goodim et al. (1999) e Bristow e Campbel (1984) a linha de tendência dos dados atravessa a reta 1:1 mudando o comportamento da estimativa da Rs de superestimada para subestimada. Essa mudança de comportamento da linha de tendência também foi observada por Da Silva et al. (2012) para o modelo $\mathrm{BC}$ para a região de Minas Gerais. 


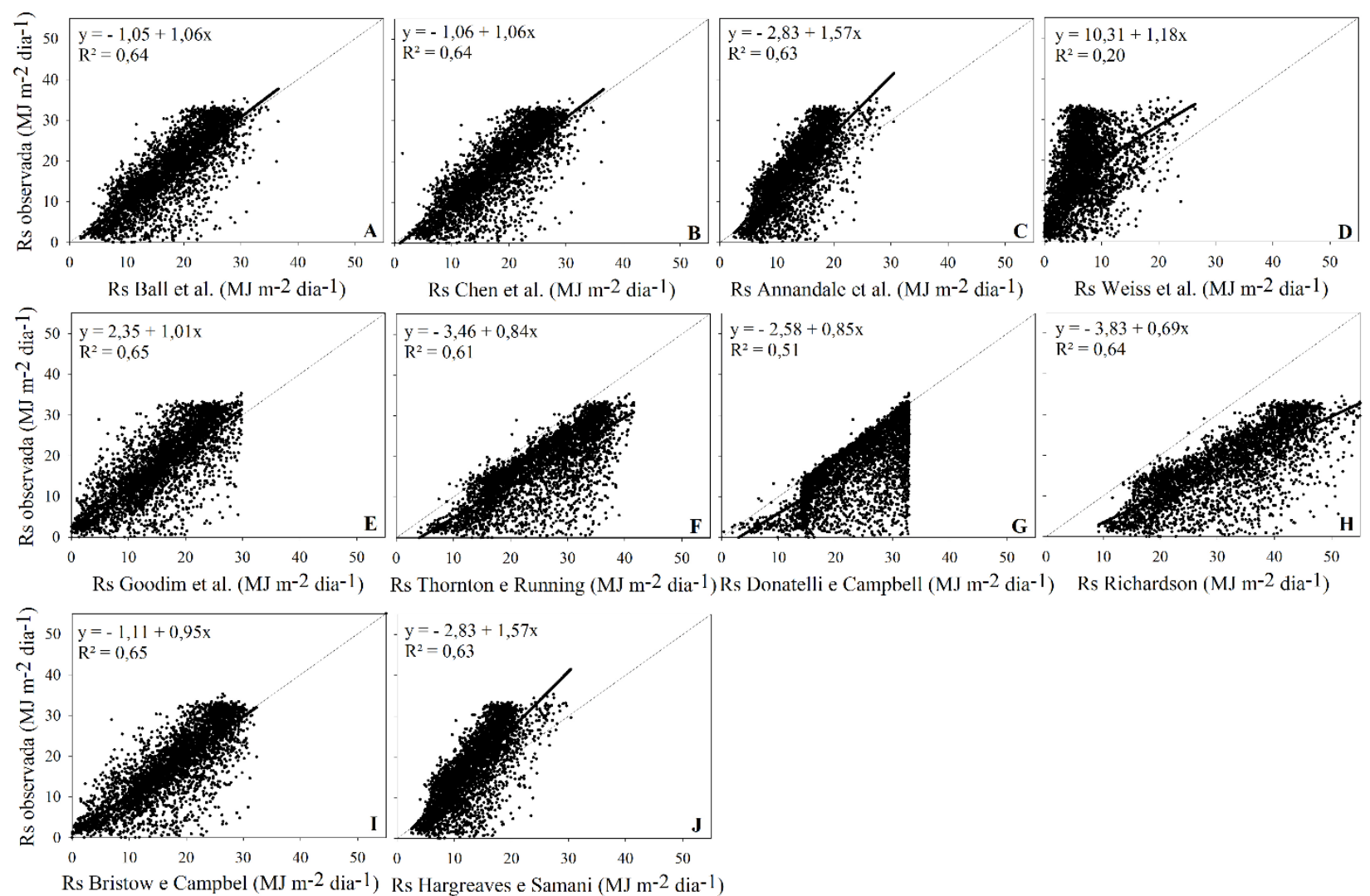

Figura 3. Representação gráfica da estimativa da radiação solar global (Rs) observada através dos dados da estação meteorológica, comparada com os valores de Rs obtidos pelos modelos de Ball et al. (2004) (1A), Chen et al. (2004) (1B), Annandale et al. (2002) (1C), Weiss et al. (2001) (1D), Goodim et al. (1999) (1E), Thornton e Running, (1999) (1F), Donatelli e Campbell (1998) (1G), Richardson (1985) (1H), Bristow e Campbel (1984) (1I) e Hargreaves e Samani (1982) (1J), para o município de São Borja-RS, para o período de 2007 a 2016.

Analisando a dispersão dos pontos nas representações gráficas para o município de São Borja (Figura 3), observa-se que modelos de Ball, Chen, Goodim et al. (1999) e Bristow e Campbel (1984) apresentaram ajuste satisfatório, apesar de seus $\mathrm{R}^{2}$ variarem em torno de 0,64, com a linha de tendência dos dados muito próxima a reta $1: 1$.

Os modelos de Ann e HS apresentaram $\mathrm{R}^{2}$ de 0,63 , semelhante aos modelos citados acima, no entanto, conforme a dispersão dos pontos, observou-se uma tendência de subestimação da Rs. Já os modelos Thornton e Running, (1999), Donatelli e Campbell (1998) e Richardson (1985) apresentaram tendência de superestimar a Rs, concentrando os pontos abaixo da reta $1: 1$. O modelo proposto por Weiss et al. (2001) não se ajustou aos dados de São Borja, pois o comportamento da linha de tendência não representou a dispersão dos dados e seu $\mathrm{R}^{2}$ foi de 0,20 .
A tendência de subestimativa do modelo WS também foi observada por Borges et al. (2010), em estudo realizado em Cruz das Almas - BA. Os referidos autores também observaram a inadequação do modelo para a estimativa da Rs para aquela localidade, com valores de EAM e RQME muito superiores aos demais modelos estudados, resultado também observado no presente trabalho, atribuindo esse desempenho a obtenção de valores baixos de transmissividade atmosférica através da equação do modelo.

Através de uma análise geral dos três municípios da região estudada, observou-se o melhor desempenho dos modelos Ball e Chen, que requereram a calibração de coeficientes. Segundo Weiss e Hays (2004), ajustar ou calibrar os coeficientes dos modelos empíricos para cada cidade ou região em específico implica encontrar um coeficiente empírico que melhor se ajusta às condições ambientais e que 


\section{ESTIMATIVA DA RADIAÇÃO SOLAR GLOBAL PARA A REGIÃO FRONTEIRA OESTE DO RIO GRANDE DO SUL}

reduz o erro médio das diferenças entre valores estimados e observados da radiação solar.

No presente trabalho, observou-se que a calibração com dados locais resultou em estimativas mais precisas da Rs com os modelos Ball e Chen, porém o mesmo não ocorreu para os modelos HS-R, que resultou na superestimava da radiação e $\mathrm{BC}$ que apresentou desempenho bom, porém inferior aos de Ball e Chen e equivalente aos modelos sem coeficientes calibrados.

O modelo HS-R é uma adaptação de Richardson ao modelo de HS. No modelo original de HS o coeficiente Krs proposto pelos autores varia de 0,17 a 0,19 , e este coeficiente multiplica o restante da equação. Já para o modelo proposto por Richardson, o coeficiente Krs foi substituído pelos coeficientes " $a$ " e " $b$ " sendo "a" uma variável independente. No presente trabalho os valores ajustados para o coeficiente "b" variaram de 0,25 a 0,30 entre os municípios estudados, valores estes que são superiores aos propostos por HS em sua equação original. $\mathrm{O}$ fato de que o coeficiente "b" ainda é somado ao coeficiente "a", é mais um fator que explica a superestimação do modelo HS-R para este estudo.

Em relação aos modelos sem necessidade de coeficiente de calibração, observou-se um desempenho satisfatório dos modelos Ann, Gd e HS, apresentando menor variação nos parâmetros de desempenho em relação aos modelos Ball, Chen e BC. Devido a maior simplicidade dos primeiros (Ann, Gd e HS), estes modelos são uma alterativa viável para estimar a Rs quando não é possível a obtenção de dados medidos de Rs para calibração.

Os valores de $R^{2}$ variando entre $0,63 \mathrm{e}$ 0,74 para os modelos que se adequaram a região da Fronteira Oeste, resultados, estes, indicados como satisfatórios para a estimativa da Rs, segundo Borges et al. (2010).

No estado de Mato Grosso Souza et al. (2017) também encontraram coeficientes de determinação muito próximos e esses variando entre 0,60 e 0,75 .

Os valores do $\mathrm{R}^{2}$ mais distantes a $1 \mathrm{e}$ demais parâmetros de desempenho de todos os modelos estudados podem ser atribuídos ao fato de que estes baseiam suas estimativas na radiação solar no topo da atmosfera e na amplitude térmica. Suas equações pressupõem que a diferença na temperatura máxima e mínima está diretamente relacionada à fração de radiação extraterrestre recebida no nível do solo.

No entanto, existem outros fatores, que não são incluídos nas equações, que podem influenciar a diferença de temperatura. Esses fatores incluem nebulosidade, umidade, latitude, elevação, topografia ou proximidade a um grande corpo hídrico (BENGHANEM e MELLIT, 2014).

\section{CONCLUSÕES}

Os modelos de Ball et al. (2004) e Chen et al. (2004), com os coeficientes calibrados, dentre os demais avaliados, são os que melhor estimam a radiação solar global (Rs) para os municípios de Alegrete, Uruguaiana e São Borja, estudados nesse trabalho.

Recomenda-se a utilização dos modelos Annandale et al. (2002) e Goodim et al. (1999) entre aqueles que não necessitam de coeficientes de ajuste em suas equações para estimativa da Rs para região de estudo.

Devido ao baixo desempenho observado para os modelos de Weiss et al. (2001), Thornton e Running, (1999), Donatelli e Campbell (1998), Richardson (1985) e Bristow e Campbel (1984), não se recomenda a utilização dos mesmos para a região da Fronteira Oeste do Rio Grande do Sul.

\section{RERERÊNCIAS BIBLIOGRAFICAS}

ALLEN, R.G.; PEREIRA, L.S.; RAES, D.;
SMITH, M. Crop evapotranspiration:
guidelines, for computing crop water requirements.
Irrigation,and Drainage Paper 56. , p.300, 1998.

ALMOROX, J.; HONTORIA, C.; BENITO, M. Models for obtaining daily global solar radiation with measured air temperature data in Madrid (Spain). Applied Energy, v.88, p.1703-1709, 2011. 
https://doi.org/10.1016/j.apenergy.2010.11.00 $\underline{3}$.

ANNANDALE, J.; JOVANOVIC, N.; BENADE, N.; ALLEN, R. Software for missing data error analysis of PenmanMonteith reference evapotranspiration. Irrigation Science, v.21, n.2, p.57-67, 2002. https://doi.org/10.1007/s002710100047.

AL-SALAYMEH, A. Model for the prediction of global daily solar radiation on horizontal surfaces for Amman city. Emirates Journal of Engineering Research, v.11, p.49-56, 2006.

ALVARES, C. A.; STAPE, J. L.; SENTELHAS, P. C.; DE MORAES, G.; LEONARDO, J.; SPAROVEK, G. Köppen's climate classification map for Brazil. Meteorologische Zeitschrift, v. 22, p. 711-728, 2013. https://doi.org/10.1127/09412948/2013/0507.

BALL, R.A.; PURCELL, L.C.; CAREY, S.K. Evaluation of solar radiation prediction models in North America. Agronomy Journal, Wisconsin, v.96, n.2, p.391-397, 2004.

BENGHANEM, M.; MELLIT, A. A simplified calibrated model for estimating daily global solar radiation in Madinah, Saudi Arabia. Theoretical and applied climatology, v. 115, n. $1-2, \quad$ p. 197-205, 2014. https://doi.org/10.1007/s00704-013-0884-2.

BEUTLER, A.N.; MUNARETO, J, D.; RAMÃO, C. J.; GALON, L.; DIAS, N. P.; POZZEBON, B. C.; RODRIGUES, L. A. T.; MUNARETO, G. S.; GIACOMELI, R.; RAMOS, P. V. Propriedades físicas do solo e produtividade de arroz irrigado em diferentes sistemas de manejo. Revista Brasileira de Ciência do Solo. Viçosa, v. 36, p:1601-1607 2012.

BORGES, V. P.; de OLIVEIRA, A. S.; MAURÍCIO FILHO, A. C.; da SILVA, T. S.; PAMPONET, B. M. Avaliação de modelos de estimativa da radiação solar incidente em Cruz das Almas, Bahia. Revista Brasileira de
Engenharia Agrícola e Ambiental, v.14, n.1, p.74-80, 2010.

BRISTOW, K.L.; CAMPBELL, G.S. On the relationship beetween incoming solar radiation and daily maximum and minimum temperature. Agricultural and Forest Meteorology, Pullman, v.31, n.2, p.159-166, 1984.

BURIOL, G. A.; ESTEFANEL, V.; HELDWEIN, A. B.;PRESTES, S.D.; Horn, J. F. C. Estimativa da radiação solar global a partir dos dados de insolação, para Santa Maria RS.

Ciência Rural, v. 42, p. 1563-1567, 2012.

CHEN, R.S.; ERSI, K.; YANG, J.P.; LU, S.H.; ZHAO, W.Z. Validation of five global radiation models with measured daily data in China. Energy Conversion and Management, v.45, n.11, p.1759-1769, 2004.

CONAB - COMPANHIA NACIONAL DE ABASTECIMENTO. Acompanhamento da safra brasileira de grãos - safra 2017/18 v5 n.7, sétimo levantamento, abril 2018. Brasília: CONAB. Disponível em: < http://www.conab.gov.br >. Acesso em: 8 mai. 2018.

DAL PAI, E.; ESCOBEDO, J. F. Estimativa da radiação atmosférica em função dos índices radiométricos kt e kd para Botucatu-SP. Energia na Agricultura, v. 30, n. 2, p. 172179, 2015. http://dx.doi.org/10.17224/EnergAgric.2015v3 On2p172-179.

da SILVA, V. J.; da SILVA, C. R.; RESENDE FINZI, R.; da SILVA DIAS, N. Métodos para estimar radiação solar na região noroeste de Minas Gerais. Ciência Rural, v. 42, n. 2, 2012.

DONATELLI, M.; CAMPBELL, G. S. A simple model to estimate global solar radiation. In: In: ZIMA, M.; BARTOSOVA, M.L., Eds., Proceedings of the Fifth European Society of Agronomy Congress, Nitra. 1998. p. 133-134. Disponivel em: <https://www.scirp.org/(S(oyulxb452alntlaej1 
nfow45))/reference/ReferencesPapers.aspx?Re ferenceID $=2145684>$. Acesso em: 7 mai. 2018.

GOODIM, D.G.; HUTCHINSON, J.M.S.; VANDERLIP, R.L.; KNAPP, M.C. Estimating solar irradiance for crop modeling using daily air temperature data. Agronomy Journal, Wisconsim, v.91, n.5, p.845-851, 1999.

HARGREAVES, G.H.; SAMANI, Z.A. Estimating potential evapotranspiration. Journal of Irrigation and Drainage Engeneering, v.108, n.3, p.225- 230, 1982.

IRGA- Instituto Rio Grandense do Arroz. Safras: Produção Municipal 2016/17. Porto Alegre: IRGA. Disponível em: < http://irga.rs.gov.br/safras-2 >. Acesso em: 10 jul. 2018.

MACÊDO, K. G.; OLIVEIRA, J. B.; ARRAES, F. D. D.; TORRES, W. L. V.; JUNIOR, J. C. L. Estimativa da radiação solar global com dados de temperatura do ar em seis cidades do estado do Ceará. Engenharia na agricultura, v. 24, n. 1, p. 68-75, 2016. https://doi.org/10.13083/reveng.v24i1.612.

RICHARDSON, C.W. Weather simulation for crop management models. Transaction ASABE, v.28, n.5, p.1602-1606, 1985.

SOUZA, A. P. D.; SILVA, A. C. D.; TANAKA, A. A.; ULIANA, E. M.; ALMEIDA, F. T. D.; KLAR, A. E.; GOMES, A. W. A. Global radiation by simplified models for the state of Mato Grosso, Brazil. Pesquisa agropecuária brasileira. v. 52, n. 4, p. $215-227$, abr. 2017. http://dx.doi.org/10.1590/s0100204x2017000400001

STEINMETZ, S.; BRAGA, H.J. Zoneamento de arroz irrigado por épocas de semeadura nos estados do Rio Grande do Sul e de Santa Catarina. Revista Brasileira de Agrometeorologia, v.9, n.3, p.429-438, 2001.

STEINMETZ, S.; DE ASSIS, F. N.; BURIOL, G. A.; ESTEFANEL, V.; AMARAL, A. G.; FERREIRA, J. S. A. Mapeamento das probabilidades de ocorrência de temperatura mínimas do ar, durante o período reprodutivo do arroz irrigado, no Estado do Rio Grande do Sul. Revista Brasileira de Agrometeorologia, v. 11, n.1, p. 169-179, 2003.S

TEINMETZ, S.; NUNES A. D.; BAPTISTA J. S., Estimativa da produtividade de arroz irrigado em função da radiação solar global e da temperatura mínima do ar. Ciência Rural, v.43, n.2, p.206-211, 2013.

THORNTON, P. E.; RUNNING, S. W. An improved algorithm for estimating incident daily solar radiation from measurements of temperature, humidity and precipitation. Agricultural and Forest Meteorology, v.93, n.4, p.211-228, 1999.

WEISS, A.; HAYS, C. J.; HU, Q.; EASTERLING, W. E. Incorporating bias error in calculating solar irradiance: implications for crop simulations. Agronomy Journal, v.93, n.6, p.1321-1326, 2001.

WEISS, A.; HAYS, C. J. Simulation of daily solar irradiance. Agricultural and Forest Meteorology, v.123, n.3-4, p.187-199, 2004. 